\title{
Complications of Common Gynecologic Surgeries among HIV-Infected Women in the United States
}

\author{
Ana Penman-Aguilar, ${ }^{1}$ Maura K. Whiteman, ${ }^{1}$ Shanna Cox, ${ }^{1}$ Samuel F. Posner, ${ }^{1}$ \\ Susan F. Meikle, ${ }^{2}$ Athena P. Kourtis, ${ }^{1}$ and Denise J. Jamieson ${ }^{1}$ \\ ${ }^{1}$ National Center for Chronic Disease Prevention and Health Promotion, Centers for Disease Control and Prevention, \\ 4770 Buford Highway-Mailstop K-34, Atlanta, GA 30341, USA \\ ${ }^{2}$ The Eunice Kennedy Shriver National Institute of Child Health and Human Development, National Institutes of Health, \\ Building 31, Room 2A32, MSC 2425, 31 Center Drive, Bethesda, MD 20892, USA
}

Correspondence should be addressed to Ana Penman-Aguilar, bpv4@cdc.gov

Received 31 January 2012; Revised 12 March 2012; Accepted 16 March 2012

Academic Editor: Gregory T. Spear

Copyright (๑) 2012 Ana Penman-Aguilar et al. This is an open access article distributed under the Creative Commons Attribution License, which permits unrestricted use, distribution, and reproduction in any medium, provided the original work is properly cited.

\begin{abstract}
Objective. To compare frequencies of complications among HIV-infected and-uninfected women undergoing common gynecological surgical procedures in inpatient settings. Methods. We used 1994-2007 data from the Nationwide Inpatient Sample of the Healthcare Cost and Utilization Project, a nationally representative sample of inpatient hospitalizations. Our analysis included discharge records of women aged $\geq 15$ undergoing hysterectomy, oophorectomy, salpingectomy for ectopic pregnancy, bilateral tubal sterilization, or dilation and curettage. Associations between HIV infection status and surgical complications were evaluated in multivariable logistic regression models, adjusting for key covariates. Results. For each surgery, HIV infection was associated with experiencing $\geq 1$ complication. Adjusted ORs ranged from 2.0 (95\% confidence interval $(\mathrm{CI}): 1.7,2.2)$ for hysterectomy with oophorectomy to 3.1 (95\% CI: 2.4, 4.0) for bilateral tubal sterilization with no comorbidity present. HIV infection was positively associated with extended length of stay and infectious complications of all of the surgeries examined. For some surgeries, it was positively associated with transfusion and anemia due to acute blood loss. Among HIV-infected women, the odds of infectious and other complications did not decrease between 1994-2000 and 2001-2007. Conclusion. HIV infection was associated with elevated frequencies of complications of gynecologic surgeries in the US, even in the era of HAART.
\end{abstract}

\section{Introduction}

Until recently, most general obstetrician-gynecologists in the United States (US) had little opportunity to provide care to HIV-infected women. Screening guidelines published in 2006 and $2008[1,2]$ and advances in treatment have changed the landscape of treatment and care of HIV-infected women. As more women test positive for HIV and as HIVinfected women live longer, healthier lives [3], increasing numbers of women of reproductive age will be living with an HIV diagnosis, and general obstetrician-gynecologists will increasingly encounter women who have been diagnosed with HIV infection. Recognizing the increased need for information and guidance for providing optimal gynecologic care for HIV-infected women, the American College of
Obstetricians and Gynecologists recently published a practice bulletin on the topic [4].

The literature on the risk of postoperative complications among HIV-infected patients is mixed. For example, a large retrospective study of surgical outcomes that used data from the Kaiser Permanente Medical Care Program revealed greater 12-month mortality and greater incidence of post-operative pneumonia; however, no other post-operative complications were elevated among HIV-infected patients [5]. Notably, despite HIV-infected women having an elevated risk for some conditions leading to gynecologic surgery [610], little systematic evidence is available on the risk of complications of gynecologic surgeries among HIV-infected women. The few reports that exist are inconsistent in their findings with one study observing a significantly higher 
rate of complications among HIV-infected women [11] and two other studies yielding null results $[12,13]$. The objective of this analysis is to add to the knowledge base by describing complications among HIV-positive women undergoing certain common gynecological surgeries. We used national data from the US to compare frequencies of complications among HIV-infected and-uninfected women undergoing common gynecological surgical procedures in inpatient settings.

\section{Materials and Methods}

We used data from the Nationwide Inpatient Sample (NIS) from 1994-2007. We included several years of data in order to have a sufficient number of discharge records among HIVinfected women to create reliable estimates for selected surgical procedures. The Healthcare Cost and Utilization Project (HCUP) includes databases and software tools developed through a partnership among private industry, states, and the federal government. The NIS, the largest all-payer database of inpatient stays in the United States, is a key component of HCUP. The NIS incorporates data from approximately 8 million hospital stays per year, and it approximates a $20 \%$ stratified sample of community hospitals in the US (AHRQ, 2010). Sampling is stratified on location (rural or urban), hospital size, region of the country, teaching status, and type of ownership (public or private). As of 2007, 40 states contributed data to the NIS, and hospitals in the sampling frame comprised approximately $90 \%$ of US hospital discharges [14].

We analyzed discharge records from women aged 15 and older, excluding hospitalizations that included delivery (International Classification of Diseases, 9th revision, Clinical Modification (ICD-9-CM) codes 650; V27). We further limited the study population to include only hospitalizations during which one of the following procedures had been performed: oophorectomy (procedure codes 65.3, 65.31, $65.39,65.4,65.41,65.49,65.5,65.51,65.52,65.53,65.54$, $65.6,65.61,65.62,65.63$, and 65.64), salpingectomy for ectopic pregnancy (procedure code 66.62), bilateral tubal sterilization (procedure codes 66.2, 66.21, 66.22, 66.29, $66.3,66.31,66.32$, and 66.39), dilation and curettage (procedure codes 69.0, 69.01, 69.02, and 69.09) or hysterectomy. Hysterectomy was defined as abdominal hysterectomy (68.3, 68.39, 68.4, and 68.49), vaginal hysterectomy (68.5; $68.59)$, or total laparoscopic hysterectomy/laparoscopicassisted hysterectomy $(68.31,68.41$, and 68.51). Any type of hysterectomy with the code 54.21 was also coded as laparoscopic-assisted. We focused on these gynecologic surgical procedures because they were the most common (at least 150 surgeries performed) among hospitalizations of HIV-infected women in our dataset. With the exception of hysterectomy with concomitant oophorectomy, we excluded hospitalizations during which multiple gynecologic surgeries were performed. The NIS does not include patient identifiers, and the unit of analysis is the hospital discharge record. Although some patients may have been admitted multiple times during the study period for procedures we examined, we expect this to be rare.
Our primary outcome, experiencing at least one complication of surgical procedures, was defined as experiencing extended length of stay; transfusion; anemia due to acute blood loss; accidental puncture or laceration during a procedure; hemorrhage, hematoma, or seroma complicating a procedure; urinary tract infection; fever; other postoperative infection; urinary tract complications including urinary retention and ureteral obstruction; paralytic ileus; any of several less common complications (e.g., thromboembolism and postoperative shock). Extended length of stay was defined as being at or above the 90th percentile for that specific surgical procedure. This was equivalent to $\geq 5$ days for hysterectomy with oophorectomy, $\geq 4$ for hysterectomy alone, $\geq 9$ for oophorectomy alone, $\geq 4$ for salpingectomy for ectopic pregnancy, $\geq 5$ for bilateral tubal sterilization, and $\geq 6$ for dilation and curettage. Other complications were defined based on relevant ICD- 9 codes.

Our primary independent variable was HIV status (ICD9-CM codes 042, 043, 044, 079.53, 279.10, 279.19 795.71, 795.8 , and V08). We defined comorbidity as presence of $\geq 1$ of the following conditions/behaviors that could put women at increased risk for complications of the gynecologic surgeries we examined: obesity, diabetes, cardiac condition or hypertension, anemia, gastrointestinal ulcers, smoking, and alcohol or substance abuse. Based on review of the literature, we selected relevant ICD-9 codes for these conditions/behaviors, and we defined them accordingly.

We compared discharge records of HIV-infected and -uninfected women undergoing the gynecologic surgeries we examined on various descriptive characteristics of patients and hospitals, including age, primary payer, hospital teaching status/location, hospital region, and presence of any comorbidity. Race was not examined because some states do not report race/ethnicity data, and, among states that do report this, there are often inconsistencies and missing values in the data. Comparisons were evaluated with chi-squared tests (alpha $=0.05)$.

For each surgery, we used multivariable logistic regression to estimate the association between HIV infection status and experiencing $\geq 1$ complication of surgery, adjusting for patient age, primary payer, year of hospitalization, and presence of any comorbidity. Because of the possibility that associations between HIV infection status and the occurrence of complications might differ depending on whether comorbidity was present, we tested for interaction between HIV infection status and presence of any comorbidity. Associations for which statistically significant interaction was detected (alpha $=0.05$ ) are presented separately for women with and without comorbidity. In addition, we conducted multivariable logistic regression to estimate the association between HIV infection and the 4 most common complications in our sample. These included extended length of stay, transfusion, anemia due to blood loss, and all infectious complications combined (i.e., experience of urinary tract infection; fever; other postoperative infection; or contaminated or infected blood, other fluid, drug, or biological substance). Again, we adjusted models for patient age, primary payer, year of hospitalization, and presence of comorbidity, and we tested for interaction between HIV 
infection and any comorbidity. Finally, for each surgery we examined, we tested for major shifts over time by using multivariable logistic regression (with adjustment for the same variables), to compare, for hospitalizations among HIV infected women, the odds of extended length of stay, infectious complications, and all other complications combined during the time periods preceding (1994-2000) and during (2001-2007) widespread implementation of highly active antiretroviral treatment (HAART) in the US.

We used SAS-callable SUDAAN 9.0 software (RTI International, Research Triangle, Durham, NC, USA) to account for the multistage probability sampling design. All results are based on weighted estimates of hospitalizations in the US during the period of study. In 1998, the NIS sample design changed to better reflect the population of hospitals in the sample. Specifically, short-term rehabilitation hospitals were excluded, stratification variables were redefined, the discharge definition was changed, and previous-year NIS hospitals were no longer given sampling precedence. To account for the change in sample design, we applied an alternate set of NIS discharge and hospital weights (based on the 1998 design) to 1994-1997 data [15]. All programming was independently duplicated by a second data analyst. Because the study utilized deidentified data from a publicly available data set, the Centers for Disease Control and Prevention determined that human-subject research review was not required.

\section{Results}

During the years 1994-2007, there were an estimated $14,922,397$ surgeries of interest (hysterectomy, oophorectomy, salpingectomy for ectopic pregnancy, bilateral tubal sterilization, or dilation and curettage) among women aged 15 and older. Excluding delivery hospitalizations $(n=$ $4,303,344)$ and hospitalizations during which more than one of the gynecologic surgeries we examined were performed $(n=1,682,573)$ resulted in an analytic sample of an estimated $8,939,780$ surgeries, 18,177 of which were among HIV-infected women, and 8,921,603 of which were among HIV-uninfected women.

The distribution of type of gynecological surgery differed among hospitalizations of HIV-infected and-uninfected women, with bilateral tubal sterilization ( $12.6 \%$ versus $3.4 \%)$ and dilation and curettage (12.7\% versus $6.8 \%)$ being more common among HIV-infected women in our sample (Table 1). For hospitalizations that included hysterectomy, the distribution of surgical approach also varied according to the woman's HIV infection status. Regardless of whether an oophorectomy was performed concomitantly, HIV-infected women in our sample more often underwent abdominal hysterectomies $(87.0 \%$ versus $82.6 \%$ for hysterectomy with oophorectomy and $61.8 \%$ versus $55.9 \%$ for hysterectomy alone). Laparoscopic hysterectomy was performed less often among HIV-infected women (9.3\% versus $11.1 \%$ for hysterectomy with oophorectomy and $8.3 \%$ versus $12.7 \%$ for hysterectomy alone). Compared to HIV-uninfected women, HIV-infected women hospitalized for gynecological surgeries were more often under 35 years of age (34.6\% versus $20.6 \%$ ), less often relied on private insurance as the primary payment source $(29.1 \%$ versus $69.6 \%)$, more often received care in urban teaching hospitals (67. $7 \%$ versus $42.5 \%$ ) and in the southern or northeastern US (83.7\% versus $57.6 \%)$. HIVinfected women more often presented with comorbidity (44.7\% versus $34.1 \%)$.

For hysterectomy with or without oophorectomy, oophorectomy without hysterectomy, salpingectomy, and dilation and curettage, HIV infection was positively associated with experiencing $\geq 1$ complication of surgery in models adjusted for age, primary payer, year, and presence of comorbidity (Table 2). However, for bilateral tubal sterilization, the association between HIV infection status and experiencing $\geq 1$ complication differed according to whether comorbidity was present (interaction $P$ value $<0.001$ ), and HIV infection was positively associated with experiencing complications only among women without comorbidity. The magnitude of the association between HIV infection and experiencing $\geq 1$ complication ranged from two-fold for hysterectomy with oophorectomy (adjusted odds ratio (aOR): 2.0; 95\% confidence interval $(\mathrm{CI}): 1.7,2.2)$ to more than three-fold for bilateral tubal sterilization in the absence of comorbidity (aOR: 3.1; 95\% CI: 2.4, 4.0).

When we examined the effect of HIV infection on the occurrence of common specific complications, we found that HIV infected women were more likely than uninfected women to experience infectious complications of all the gynecologic surgeries we examined (Table 3 ). They also more often experienced an extended length of stay following these surgeries. However, for bilateral tubal sterilization, the association between HIV infection and extended length of stay differed by the presence of comorbidity (interaction $P$ value $<0.001)$; HIV-infected women more often experienced extended length of stay only when comorbidity was absent. For the following types of surgery, transfusion was more often performed on HIV-infected women than HIVuninfected women: hysterectomy without oophorectomy, bilateral tubal sterilization, and dilation and curettage. For dilation and curettage, but not for the other types of gynecologic surgeries we examined, HIV-infected women more often experienced anemia due to acute blood loss.

Among hospitalizations of HIV-infected women, there were no statistically significant changes from 1994-2000 to 2001-2007 in the frequency of any of the three groups of complications we examined for changes over time (extended length of stay, infectious complications, and all others combined).

\section{Discussion}

HIV infection was associated with elevated frequencies of surgical complications of the gynecologic surgeries we examined. Not only was positive HIV status associated with experiencing any of several surgical complications, but it also showed higher frequencies of specific complications such as needing to undergo transfusion. In most cases, the presence of comorbidity did not alter the association between HIV and surgical complications. Overall, we observed an increased 
TABLE 1: Demographic and hospitalization characteristics, surgical procedure and approach, and presence of comorbidity among selected gynecological surgeries, by HIV status (United States, 1994-2007).

\begin{tabular}{|c|c|c|c|c|c|}
\hline \multirow{2}{*}{$\begin{array}{l}\text { Characteristic } \\
\text { Patient age }\end{array}$} & \multicolumn{2}{|c|}{$\begin{array}{c}\text { HIV-Infected } \quad N=18,177 \\
n \text { percentage }\end{array}$} & \multicolumn{2}{|c|}{$\begin{array}{c}\text { HIV-Uninfected } N=8,921,603 \\
n \text { percentage }\end{array}$} & \multirow[t]{2}{*}{$P$ value } \\
\hline & & & & & \\
\hline $15-34$ & 6297 & 34.64 & 1836185 & 20.58 & \multirow{4}{*}{$<0.001$} \\
\hline $35-44$ & 7539 & 41.48 & 3235700 & 36.27 & \\
\hline $45-54$ & 3549 & 19.53 & 2361684 & 26.47 & \\
\hline $55+$ & 792 & 4.36 & 1488034 & 16.68 & \\
\hline \multicolumn{6}{|l|}{ Any comorbidity* } \\
\hline Yes & 8123 & 44.69 & 3040273 & 34.08 & $<0.001$ \\
\hline \multicolumn{6}{|l|}{ Primary payer** } \\
\hline Medicare & 2109 & 11.64 & 939970 & 10.57 & \multirow{4}{*}{$<0.001$} \\
\hline Medicaid & 8255 & 45.55 & 1040880 & 11.71 & \\
\hline Private Insurer & 5276 & 29.11 & 6190159 & 69.63 & \\
\hline Other & 2483 & 13.7 & 718531 & 8.08 & \\
\hline \multicolumn{6}{|l|}{ Hospital teaching status/location ${ }^{\dagger}$} \\
\hline Rural & 964 & 5.31 & 1349712 & 15.16 & \multirow{3}{*}{$<0.001$} \\
\hline Urban nonteaching & 4901 & 27.01 & 3772124 & 42.37 & \\
\hline Urban teaching & 12279 & 67.67 & 3781486 & 42.47 & \\
\hline \multicolumn{6}{|l|}{ Hospital region } \\
\hline Northeast & 5512 & 30.32 & 1555612 & 17.44 & \multirow{4}{*}{$<0.001$} \\
\hline Midwest & 1928 & 10.61 & 2070666 & 23.21 & \\
\hline South & 9703 & 53.38 & 3583074 & 40.16 & \\
\hline West & 1034 & 5.69 & 1712251 & 19.19 & \\
\hline \multicolumn{6}{|l|}{ Surgical procedure (approach) } \\
\hline $\begin{array}{l}\text { Hysterectomy with concomitant } \\
\text { oophorectomy }{ }^{\dagger \dagger}\end{array}$ & 6546 & 36.01 & 4314430 & 48.36 & \multirow{12}{*}{$<0.001^{\ddagger}$} \\
\hline (Abdominal) & $(5693)$ & $(86.97)$ & (3561463) & $(82.55)$ & \\
\hline (Vaginal) & $(247)$ & $(3.77)$ & $(272811)$ & $(6.32)$ & \\
\hline (Laparoscopic) & $(606)$ & $(9.26)$ & $(480157)$ & $(11.13)$ & \\
\hline Hysterectomy alone $\mathrm{t}^{\dagger \dagger}$ & 4591 & 25.26 & 2235199 & 25.05 & \\
\hline (Abdominal) & $(2836)$ & $(61.77)$ & $(1248765)$ & $(55.87)$ & \\
\hline (Vaginal) & $(1373)$ & $(29.90)$ & $(703311)$ & $(31.47)$ & \\
\hline (Laparoscopic) & $(382)$ & $(8.33)$ & $(283122)$ & $(12.67)$ & \\
\hline Oophorectomy alone & 1841 & 10.13 & 1214082 & 13.61 & \\
\hline Salpingectomy for ectopic pregnancy & 609 & 3.35 & 252689 & 2.83 & \\
\hline Bilateral tubal sterilization & 2290 & 12.60 & 302380 & 3.39 & \\
\hline Dilation and curettage & 2301 & 12.66 & 602823 & 6.76 & \\
\hline
\end{tabular}

*Including obesity, diabetes, cardiac condition or hypertension, asthma, anemia, gastrointestinal ulcers, smoking, and alcohol or substance abuse.

${ }^{* *} N=8,907,664$ due to missing values.

${ }^{\dagger} N=8,921,466$.

${ }^{\dagger} P$ value for surgical approach $<0.001$.

${ }^{\ddagger} P$ value for surgical procedure. 
TABLE 2: Estimated numbers of specific procedures, percentages with any complication, and adjusted odds ratios for experiencing at least one complication, by HIV status, for selected gynecological surgeries (United States, 1994-2007).

\begin{tabular}{|c|c|c|c|c|c|}
\hline \multirow{2}{*}{ Surgery } & \multicolumn{2}{|c|}{ HIV-Infected } & \multicolumn{2}{|c|}{ HIV-Uninfected } & \multirow{2}{*}{$\mathrm{aOR}(95 \% \mathrm{CI})^{\dagger}$} \\
\hline & $\begin{array}{l}n \text { undergoing } \\
\text { procedure }\end{array}$ & $\begin{array}{l}\text { Percentage with any } \\
\text { complication* }\end{array}$ & $\begin{array}{l}n \text { undergoing } \\
\text { procedure }\end{array}$ & $\begin{array}{l}\text { Percentage with } \\
\text { any complication }\end{array}$ & \\
\hline $\begin{array}{l}\text { Hysterectomy with concomitant } \\
\text { oophorectomy }\end{array}$ & 6546 & 37.47 & 4314430 & 24.95 & $2.0(1.7,2.2)$ \\
\hline $\begin{array}{l}\text { Hysterectomy without } \\
\text { oophorectomy }\end{array}$ & 4591 & 41.4 & 2235199 & 20.8 & $2.3(2.0,2.6)$ \\
\hline $\begin{array}{l}\text { Oophorectomy without } \\
\text { hysterectomy }\end{array}$ & 1841 & 44.64 & 1214082 & 28.33 & $2.6(2.1,3.2)$ \\
\hline $\begin{array}{l}\text { Salpingectomy for ectopic } \\
\text { pregnancy }\end{array}$ & 609 & 50.56 & 252689 & 29.93 & $2.2(1.5,3.2)$ \\
\hline \multicolumn{6}{|l|}{ Bilateral tubal sterilization ${ }^{\ddagger}$} \\
\hline Any comorbidity & 811 & 36.07 & 54514 & 31.56 & $1.2(0.9,1.7)$ \\
\hline No comorbidity & 1478 & 38.4 & 247866 & 15.97 & $3.1(2.4,4.0)$ \\
\hline Dilation and curettage & 2301 & 54.23 & 602823 & 29.64 & $2.8(2.3,3.4)$ \\
\hline
\end{tabular}

${ }^{*}$ Complications include extended length of stay; accidental puncture or laceration during procedure; hemorrhage, hematoma, or seroma complicating a procedure; anemia due to acute blood loss; transfusion; urinary tract infection; fever; other postoperative infection; urinary tract complications including urinary retention and ureteral obstruction; paralytic ileus; thromboembolism; postoperative shock; disruption of operation wound; postoperative fistula; hypotension; cardiac arrest; respiratory arrest; foreign body left during procedure; acute reaction to foreign substance accidentally left during a procedure; failure of sterile precautions during procedure; failure in dosage; mechanical failure of instrument or apparatus during procedure; contaminated or infected blood, other fluid, drug, or biological substance; removal of other organ (partial or total); nonspecified other complications of medical care; other complications of procedures.

${ }^{\dagger}$ Adjusted for patient age (modeled as continuous), primary payer (private insurance versus other), year of hospitalization (modeled as continuous), and presence of any comorbidity (obesity, diabetes, cardiac condition or hypertension, asthma, anemia, gastrointestinal ulcers, smoking, and alcohol or substance abuse).

${ }^{\ddagger}$ Results are presented separately depending on the presence of comorbidity due to statistically significant interaction between HIV status and comorbidity.

occurrence of complications among HIV-infected women undergoing gynecologic surgeries.

Comorbidity altered the association between HIV status and surgical complications of bilateral tubal sterilization. Among women who had this procedure, the frequency of experiencing at least one complication was essentially equal among HIV-infected and-uninfected women with any of the comorbidities we examined; however, when comorbidity was absent, HIV-infected women more often experienced at least one complication. We suspect that, for tubal sterilization, which is a relatively minor procedure, comorbidity may be more important than HIV status in putting women at risk of surgical complications. Nevertheless, HIV status appeared to play an important role among women without comorbidity; among these women, HIV infection was associated with increased occurrence of complications. This is consistent with the increased occurrence of complications that we observed for other surgical procedures. Notably, abdominal approaches were more often used (and laparoscopic approaches less often used) among HIV-infected women in our sample who underwent hysterectomy, as compared to uninfected women who underwent hysterectomy. This likely contributed to the increased frequency of surgical complications that we observed among HIV-infected women undergoing hysterectomy.

Little has been published on complications of gynecologic surgical procedures among HIV-infected women. A study by Grupert and colleagues included 235 gynecologic and obstetric surgeries among HIV-infected women [11]. They reported a higher complication rate among HIVinfected women, as compared to HIV-negative controls. Another study by Franz and colleagues [12] reviewed the hospital course of $24 \mathrm{HIV}$-infected patients who underwent hysterectomy and compared them to uninfected controls, finding that HIV-infected women and controls did not differ on complication rates. Sewell and colleagues reviewed 53 surgical procedures among HIV-infected women [13]. They found that HIV-infected women had approximately twice the odds of experiencing complications (17\% of HIV-infected women versus $9 \%$ of controls); however, these results did not achieve statistical significance, perhaps because of small sample size. In contrast, we examined associations between infection with HIV and frequency of complications of selected gynecologic surgeries in a large nationally representative database, and we observed statistically significant associations between HIV infection status and experiencing complications.

Our study has some limitations. We did not have information on the stage of women's HIV disease. Although frequencies of complications were higher among HIVinfected women, we could not determine the extent to which this was due to immunosuppression. Additionally, the NIS dataset includes only inpatient hospitalizations. Our findings are, therefore, only applicable to inpatient settings; results in other settings may differ. Finally, the quality of our data is dependent on the accuracy of diagnoses 
TABLE 3: Estimated percentages with specific complications* and adjusted odds ratios for experiencing complications, by HIV status, among selected gynecological surgeries (United States, 1994-2007).

\begin{tabular}{|c|c|c|c|}
\hline Surgery & $\begin{array}{c}\text { HIV-Infected } \\
(n \text { undergoing procedure }) \\
\text { percentageexperiencing complication }\end{array}$ & $\begin{array}{c}\text { HIV-Uninfected } \\
\text { ( } n \text { undergoing procedure) } \\
\text { percentage experiencing } \\
\text { complication }\end{array}$ & $\mathrm{aOR}(95 \% \mathrm{CI})^{* *}$ \\
\hline $\begin{array}{l}\text { Hysterectomy with concomitant } \\
\text { oophorectomy }\end{array}$ & $(n=6546)$ & $(n=4314430)$ & \\
\hline Extended length of stay & 25.70 & 13.25 & $2.8(2.4,3.2)$ \\
\hline Transfusion & 9.37 & 3.74 & t \\
\hline Anemia due to acute blood loss & 5.25 & 5.75 & $1.1(0.9,1.4)$ \\
\hline Infectious complications $^{\dagger \dagger}$ & 8.83 & 4.24 & $2.3(1.9,2.9)$ \\
\hline Hysterectomy without oophorectomy & $(n=4591)$ & $(n=2235199)$ & \\
\hline Extended length of stay & 31.63 & 11.45 & $2.7(2.4,3.2)$ \\
\hline Transfusion & 8.57 & 2.98 & $2.2(1.7,2.8)$ \\
\hline Anemia due to acute blood loss & 5.40 & 4.99 & $1.1(0.8,1.4)$ \\
\hline Infectious complications & 8.06 & 3.14 & $2.4(1.9,3.0)$ \\
\hline Oophorectomy without hysterectomy & $(n=1841)$ & $(n=1214082)$ & \\
\hline Extended length of stay & 23.49 & 11.91 & $4.3(3.3,5.7)$ \\
\hline Transfusion & 8.86 & 4.73 & + \\
\hline Anemia due to acute blood loss & 5.46 & 5.50 & $1.3(0.9,2.1)$ \\
\hline Infectious complications & 11.94 & 6.19 & $2.2(1.6,3.0)$ \\
\hline Salpingectomy for ectopic pregnancy & $(n=609)$ & $(n=252689)$ & \\
\hline Extended length of stay & 31.02 & 11.79 & $3.0(2.0,4.4)$ \\
\hline Transfusion & 16.48 & 9.69 & $1.7(0.9,2.9)$ \\
\hline Anemia due to acute blood loss & 13.76 & 14.36 & $1.0(0.5,1.8)$ \\
\hline Infectious complications & 8.77 & 3.07 & $2.6(1.4,4.8)$ \\
\hline Bilateral tubal sterilization & $(n=2290)$ & $(n=302380)$ & \\
\hline $\begin{array}{l}\text { Among women with any comorbidity, } \\
\text { extended length of stay }{ }^{\ddagger}\end{array}$ & 29.08 & 23.12 & $1.4(1.0,2.0)$ \\
\hline $\begin{array}{l}\text { Among women with no comorbidity, } \\
\text { extended length of stay }{ }^{\ddagger}\end{array}$ & 32.00 & 9.22 & $4.4(3.4,5.6)$ \\
\hline Transfusion & 5.01 & 1.62 & $2.3(1.5,3.6)$ \\
\hline Anemia due to acute blood loss & 3.59 & 3.73 & $0.9(0.6,1.5)$ \\
\hline Infectious complications & 7.41 & 2.57 & $2.3(1.6,3.3)$ \\
\hline Dilation and curettage & $(n=2301)$ & $(n=602823)$ & \\
\hline Extended length of stay & 27.68 & 11.04 & $3.6(2.9,4.5)$ \\
\hline Transfusion & 26.15 & 10.95 & $2.7(2.1,3.6)$ \\
\hline Anemia due to acute blood loss & 15.47 & 9.91 & $1.8(1.4,2.3)$ \\
\hline Infectious complications & 6.61 & 4.31 & $1.8(1.2,2.7)$ \\
\hline
\end{tabular}

${ }^{*}$ We present the 4 complications that were most common in our sample.

**Adjusted for patient age (modeled as continuous), primary payer (private insurance versus other), year of hospitalization (modeled as continuous), and presence of any comorbidity (obesity, diabetes, cardiac condition or hypertension, asthma, anemia, gastrointestinal ulcers, smoking, and alcohol or substance abuse).

${ }^{\dagger}$ No valid model could be generated (i.e., model did not converge).

${ }^{\dagger \dagger}$ Infectious complications included urinary tract infection; fever; other postoperative infection; and contaminated or infected blood, other fluid, drug, or biological substance.

${ }^{\ddagger}$ Results are presented separately depending on the presence of comorbidity due to statistically significant interaction between HIV status and comorbidity. 
and procedures listed in discharge records. Some of the conditions and behaviors that we classified as comorbidity may have been characterized by differential accuracy or completeness, depending on a woman's HIV status. For example, a provider may have been more or less likely to ascertain substance abuse and note it in the medical record, depending on a woman's HIV status.

Despite these limitations, our study had important strengths. It is based on a large, nationwide data set, making our findings more generalizable to inpatient gynecologic surgeries across the US. The numbers of women undergoing surgeries in our analysis exceeded those in earlier studies. The large sample size allowed for the ability to detect associations that may have been missed in other studies. Finally, unlike the earlier studies we cited, we sought to account for the relationship between HIV and comorbidity in putting women at risk for complications of gynecologic surgery.

\section{Conclusions}

In conclusion, the issue of HIV infection in gynecological treatment and care will become more prominent as more women in the US screen positive for HIV [4], and treatment adds years to women's lives [3]. Our study adds to the evidence that HIV infection is an important consideration in gynecologic treatment and care. Adequately powered prospective studies that examine factors that put HIVinfected women at higher risk of surgical complications, and that identify potentially modifiable risk factors, are needed. Results of such studies can inform development of prevention strategies to protect the health of HIV-infected women, and help reduce disparities between HIV-infected and -uninfected women in complications of gynecologic surgeries.

Our finding that the frequency of infectious and other surgical complications among hospitalizations of HIVinfected women did not decrease following widespread implementation of HAART suggests that women's access to and adherence to treatment for HIV disease could be improved. Strategies for improving access and adherence to HAART should be developed, evaluated, and prioritized. Additionally, the high proportion of HIV-infected women in our sample with comorbidities underscores the importance of health providers being well prepared to address a wide range of medical comorbidities as well as behaviors that may negatively impact the health of HIV-infected women. Treatment by multidisciplinary teams of providers may be another approach for ensuring that the health needs of HIVinfected women are addressed.

\section{Disclaimer}

The findings and conclusions in this report are those of the authors and do not necessarily represent the official position of the Centers for Disease Control and Prevention.

\section{References}

[1] B. M. Branson, H. H. Handsfield, M. A. Lampe et al., "Revised recommendations for HIV testing of adults, adolescents, and pregnant women in health-care settings," Morbidity and Mortality Weekly Report, vol. 55, no. 14, pp. 1-17, 2006.

[2] American College of Obstetricians and Gynecologists, "ACOG Committee opinion no. 410: ethical issues in genetic testing," Obstetrics \& Gynecology, vol. 111, no. 6, pp. 1495-1502, 2008.

[3] R. M. Gulick, "Antiretroviral treatment 2010: progress and controversies," Journal of Acquired Immune Deficiency Syndromes, vol. 55, no. 1, pp. S43-S48, 2010.

[4] American College of Obstetricians and Gynecologists (ACOG), "Gynecologic care for women with human immunodeficiency virus," ACOG Practice Bulletin number 117, American College of Obstetricians and Gynecologists (ACOG), Washington, DC, USA, 2010.

[5] M. A. Horberg, L. B. Hurley, D. B. Klein et al., "Surgical outcomes in human immunodeficiency virus-infected patients in the era of highly active antiretroviral therapy," Archives of Surgery, vol. 141, no. 12, pp. 1238-1245, 2006.

[6] T. V. Ellerbrock, M. A. Chiasson, T. J. Bush et al., "Incidence of cervical squamous intraepithelial lesions in HIV-infected women," Journal of the American Medical Association, vol. 283, no. 8, pp. 1031-1037, 2000.

[7] S. E. Hawes, C. W. Critchlow, M. A. Faye Niang et al., "Increased risk of high-grade cervical squamous intraepithelial lesions and invasive cervical cancer among African women with human immunodeficiency virus type 1 and 2 infections," Journal of Infectious Diseases, vol. 188, no. 4, pp. 555-563, 2003.

[8] P. Schuman, S. E. Ohmit, R. S. Klein et al., "HIV Epidemiology Research Study (HERS). Longitudinal study of cervical squamous intraepithelial lesions in human immunodeficiency virus (HIV) seropositive and at risk HIV-seronegative women," Journal of Infectious Diseases, vol. 188, pp. 128-136, 2003.

[9] L. J. Conley, T. V. Ellerbrock, T. J. Bush, M. A. Chiasson, D. Sawo, and T. C. Wright, "HIV-1 infection and risk of vulvovaginal and perianal condylomata acuminata and intraepithelial neoplasia: a prospective cohort study," Lancet, vol. 359, no. 9301, pp. 108-113, 2002.

[10] D. J. Jamieson, P. Paramsothy, S. Cu-Uvin, A. Duerr, and HIV Epidemiology Research Study Group, "Vulvar, vaginal, and perianal intraepithelial neoplasia in women with or at risk for human immunodeficiency virus," Obstetrics \& Gynecology, vol. 107, pp. 1023-1028, 2006.

[11] T. A. Grubert, D. Reindell, R. Kästner et al., "Rates of postoperative complications among human immunodefidency virus-infected women who have undergone obstetric and gynecologic surgical procedures," Clinical Infectious Diseases, vol. 34, no. 6, pp. 822-830, 2002.

[12] J. Franz, D. J. Jamieson, H. Randall, and C. Spann, "Outcomes of hysterectomy in HIV-seropositive women compared to seronegative women," Infectious Diseases in Obstetrics and Gynecology, vol. 13, no. 3, pp. 167-169, 2005.

[13] C. A. Sewell, R. Derr, and J. Anderson, "Operative complications in HIV-infected women undergoing gynecologic surgery," Journal of Reproductive Medicine for the Obstetrician and Gynecologist, vol. 46, no. 3, pp. 199-204, 2001. 
[14] Agency for Healthcare Research and Quality, Overview of the Nationwide Inpatient Sample (NIS) Healthcare Cost and Utilization Project (HCUP) http://www.hcup-us.ahrq.gov/nisoverview.jsp.

[15] R. L. Houchens and A. Elixhauser, "Using the HCUP Nationwide Inpatient Sample to estimate trends," HCUP Methods Series Report \# 2005-01. Agency for Healthcare Research and Quality, Rockville, Md, USA, 2005, http://www.hcup-us .ahrq.gov/reports/TrendReport2005_1.pdf. 


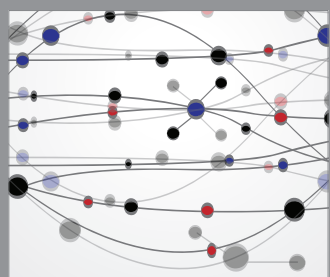

The Scientific World Journal
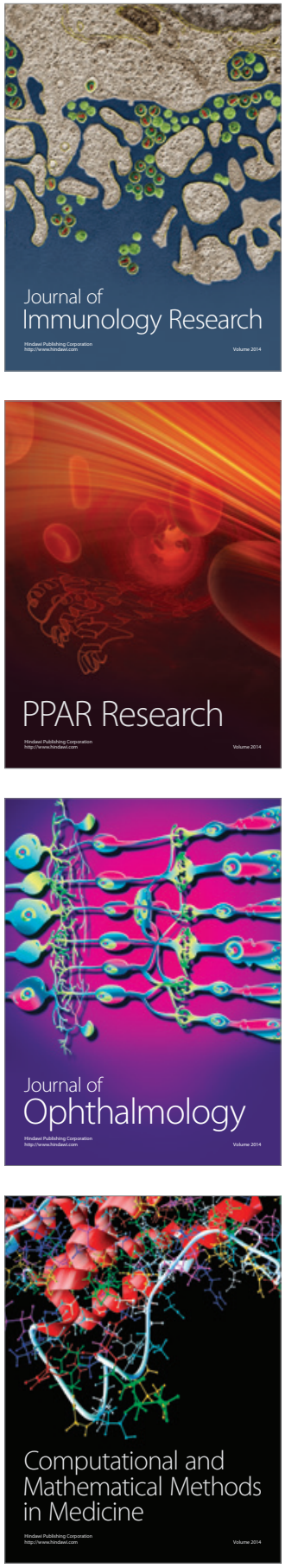

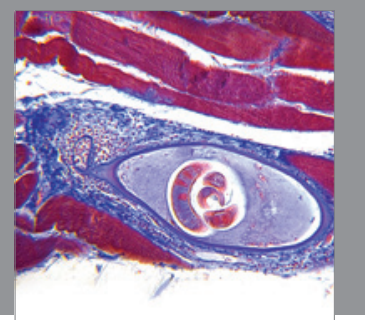

Gastroenterology

Research and Practice
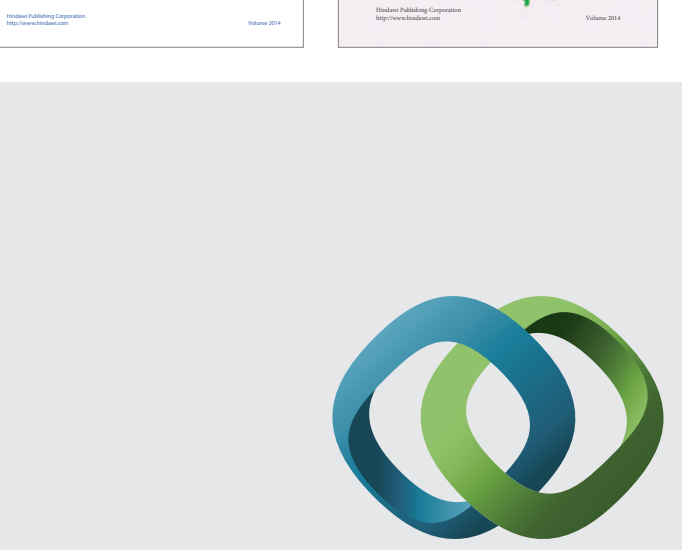

\section{Hindawi}

Submit your manuscripts at

http://www.hindawi.com
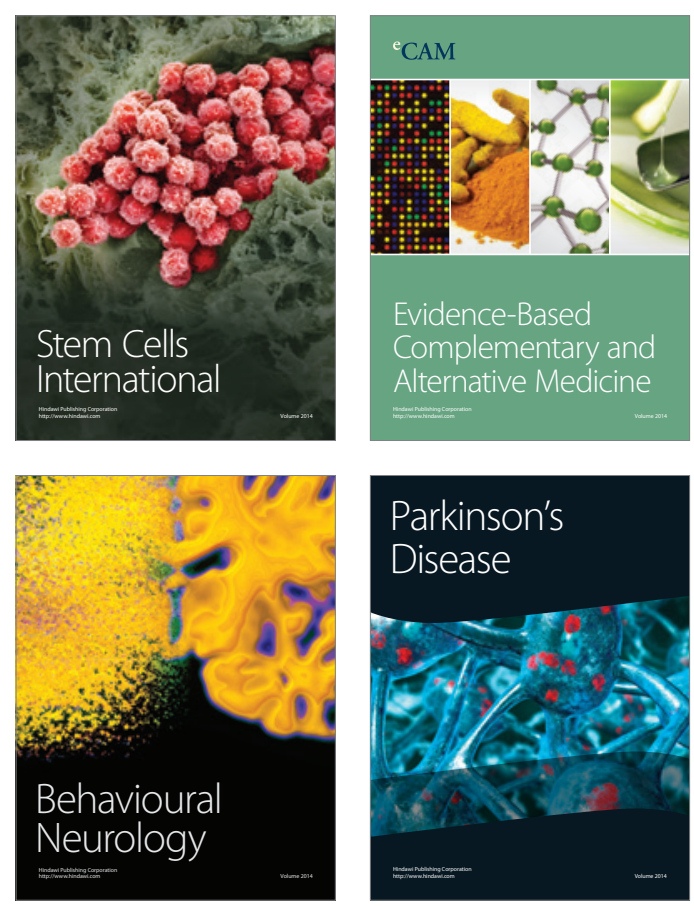

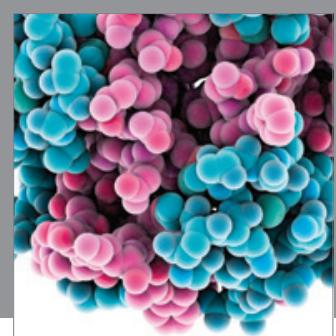

Journal of
Diabetes Research

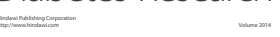

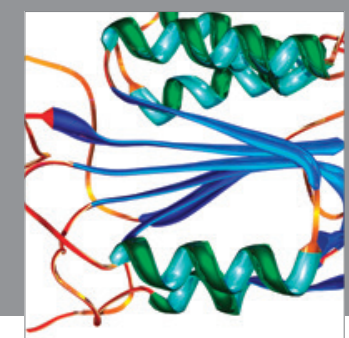

Disease Markers
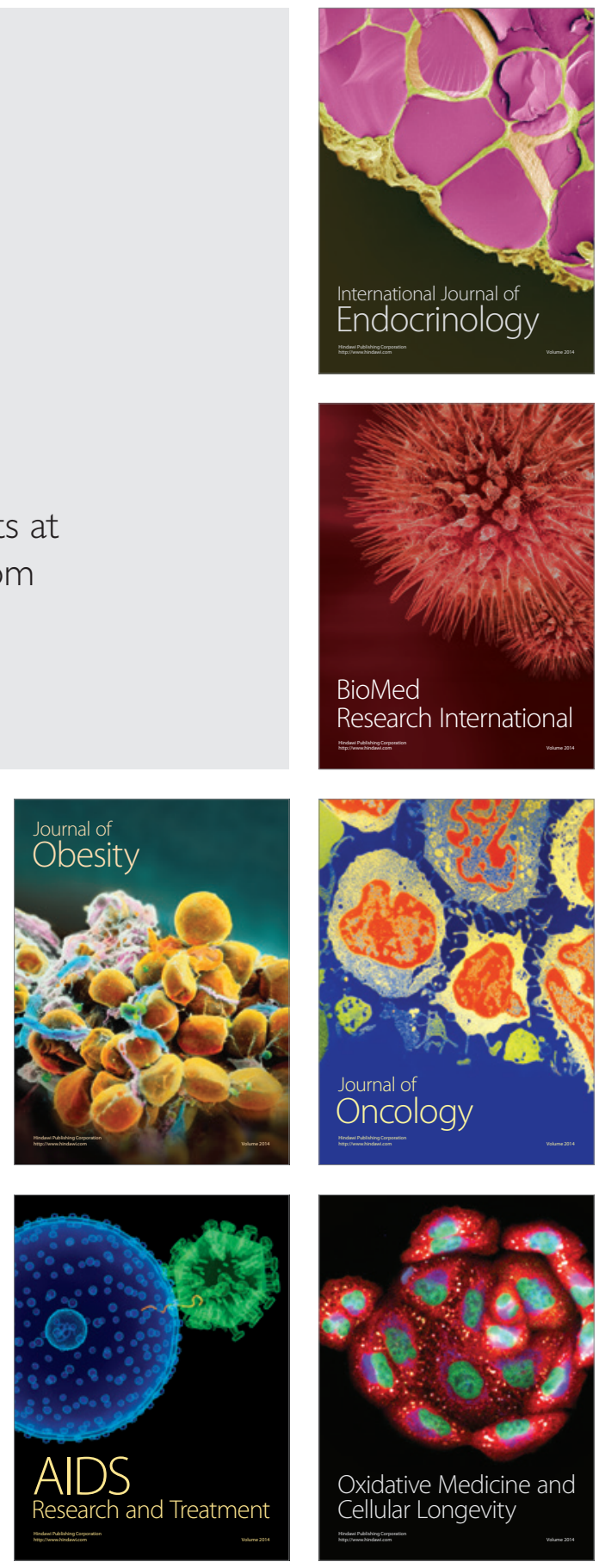